\title{
Principal Component Analyses in Anthropological Genetics
}

\author{
Xingdong Chen, Chao Chen, Li Jin \\ Ministry of Education Key Laboratory of Contemporary Anthropology, Fudan University, Shanghai, China. \\ Email: lijin.fudan@gmail.com \\ Received August 17 $7^{\text {th }}, 2011$; revised October $8^{\text {th }}, 2011$; accepted October $20^{\text {th }}, 2011$.
}

\begin{abstract}
Principal component analyses (PCA) is a statistical method for exploring and making sense of datasets with a large number of measurements (which can be thought of as dimensions) by reducing the dimensions to the few principal components (PCs) that explain the main patterns. Thus, the first PC is the mathematical combination of measurements that accounts for the largest amount of variability in the data. Here, we gave an interpretation about the principle of PCA and its original mathematical algorithm, singular variable decomposition (SVD). PCA can be used in study of gene expression; also PCA has a population genetics interpretation and can be used to identify differences in ancestry among populations and samples, through there are some limitations due to the dynamics of microevolution and historical processes, with advent of molecular techniques, PCA on Y chromosome, mtDNA, and nuclear DNA gave us more accurate interpretations than on classical markers. Furthermore, we list some new extensions and limits of PCA.
\end{abstract}

Keywords: Principal Component Analysis, Singular Value Decomposition, Human Genetics

\section{Introduction}

Principal component analysis (PCA) involves a mathematical procedure that transforms a number of possibly correlated variables into a smaller number of uncorrelated variables called principal components. The first principal component accounts for as much of the variability in the data as possible, and each succeeding component accounts for as much of the remaining variability as possible. Depending on the field of application, it is also named the discrete Karhunen-Loève transform (K.L.T.), the Hotelling transform or proper orthogonal decomposition (POD).

PCA was invented in 1901 by Karl Pearson (Pearson, 1901). Now it is mostly used as a tool in exploratory data analysis and for making predictive models. PCA involves the calculation of the eigenvalue decomposition of a data covariance matrix or singular value decomposition of a data matrix, usually after mean centering the data for each attribute. The results of a PCA are usually discussed in terms of component scores and loadings.

PCA is mathematically defined as an orthogonal linear transformation that transforms the data to a new coordinate system such that the greatest variance by any projection of the data comes to lie on the first coordinate (called the first principal component), the second greatest variance on the second coordinate, and so on. PCA is the simplest of the true eigenvector-based multivariate analyses. Often, its operation can be thought of as revealing the internal structure of the data in a way which best explains the variance in the data. If a multivariate dataset is visualised as a set of coordinates in a high-dimensional data space (1 axis per variable), PCA supplies the user with a lower-dimensional picture, a "shadow" of this object when viewed from its (in some sense) most informative viewpoint.

Luca Cavalli-Sforza and colleagues had the original insight that PCA could be applied to human genetic variation (Menozzi et al., 1978), and they eventually analyzed about 100 protein polymorphisms that had been measured in many human populations (Cavalli-Sforza et al., 1994). For several decades, PCA has been used to study human population migrations: detecting population substructure, correcting for stratification in disease studies and making qualified inferences about human history. In the recent genome wide association studies (GWAS), PCA is used to explicitly model ancestry differences between cases and controls, due to population stratification-allele frequency differences between cases and controls from systematic ancestry differences-can cause spurious associations in disease studies (Price et al., 2006). PCA is also widely used in microarray expression data analysis, to control surrogate variables, such as different studies comparison, batch effect and time course analysis (Alter et al., 2000, 2003; Alter \& Golub, 2006; Omberg et al., 2007; Yeung \& Ruzzo, 2001)

In this review, we first interpreted the principal algorithm of PCA, how it related to singular value decomposition (SVD) mathematically, and what is the difference between these two methods, in section 1; and in section 2, we discussed applications of PCA and SVD in modern genetics, such as population genetics on anthropology and illustrative gene expression applications. Finally, in section 3, we list some limit of PCA and new extensions to PCA.

\section{Section 1: Principle of PCA and SVD}

\section{Principle of PCA}

Define a data matrix, $\boldsymbol{X}^{T}$, with zero empirical mean (the empirical mean of the distribution has been subtracted from the data set), where each of the $n$ rows represents a different repetition of the experiment, and each of the $m$ columns gives a particular kind of datum. The singular value decomposition of $\boldsymbol{X}$ is $\boldsymbol{X}=\boldsymbol{W} \Sigma \boldsymbol{V}^{\boldsymbol{T}}$, where the $m \times m$ matrix $\boldsymbol{W}$ is the matrix of eigenvectors of $\boldsymbol{X} \boldsymbol{X}^{\boldsymbol{T}}$, the matrix $\boldsymbol{\Sigma}$ is an $m \times n$ rectangular diagonal matrix with nonnegative real numbers on the diagonal, and the $n \times n$ matrix $\boldsymbol{V}$ is the matrix of eigenvectors of $\boldsymbol{X}^{T} \boldsymbol{X}$. The PCA transformation that preserves dimensionality (that is, gives the same number of principal components as original variables) is then given by:

$$
Y^{T}=X^{T} W+V \Sigma^{T}
$$

Since $W$ (by definition of the SVD of a real matrix) is an orthogonal matrix, each row of $\boldsymbol{Y}^{\boldsymbol{T}}$ is simply a rotation of the corresponding row of $\boldsymbol{X}^{T}$. The first column of $\boldsymbol{Y}^{T}$ is made up of the "scores" of the cases with respect to the "principal" component, 
the next column has the scores with respect to the "second principal" component, and so on.

Given a set of points in Euclidean space, the first principal component (the eigenvector with the largest eigenvalue) corresponds to a line that passes through the mean and minimizes sum squared error with those points. The second principal component corresponds to the same concept after all correlation with the first principal component has been subtracted out from the points. Each eigenvalue indicates the portion of the variance that is correlated with each eigenvector. Thus, the sum of all the eigenvalues is equal to the sum squared distance of the points with their mean divided by the number of dimensions. PCA essentially rotates the set of points around their mean in order to align with the first few principal components. This moves as much of the variance as possible (using a linear transformation) into the first few dimensions. The values in the remaining dimensions, therefore, tend to be highly correlated and may be dropped with minimal loss of information. PCA is often used in this manner for dimensionality reduction. PCA has the distinction of being the optimal linear transformation for keeping the subspace that has largest variance. This advantage, however, comes at the price of greater computational requirement if compared, for example, to the discrete cosine transform. Non-linear dimensionality reduction techniques tend to be more computationally demanding than PCA.

\section{SVD}

This section is the most mathematically involved and can be skipped without much loss of continuity. It is presented solely for completeness. We derive another algebraic solution for PCA and in the process, find that PCA is closely related to singular value decomposition (SVD). In fact, the two are so intimately related that the names are often used interchangeably. What we will see though is that SVD is a more general method of understanding fundamental mathematical transformations. We begin by quickly deriving the decomposition. In the following section we interpret the decomposition and in the last section we relate these results to PCA.

Let $\boldsymbol{X}$ denote an $m \times n$ matrix of real-valued data and rank $r$, where without loss of generality $m \geq n$, and therefore $r \leq n$. In the case of microarray data, $x_{i j}$ is the expression level of the $i^{\text {th }}$ gene in the $j^{\text {th }}$ assay. The elements of the $i^{\text {th }}$ row of $\boldsymbol{X}$ form the $n$-dimensional vector $\boldsymbol{g}_{i}$, which we refer to as the transcriptional response of the $i^{\text {th }}$ gene. Alternatively, the elements of the $j^{\text {th }}$ column of $\boldsymbol{X}$ form the $m$-dimensional vector $\boldsymbol{a}_{j}$, which we refer to as the expression profile of the $j^{\text {th }}$ assay.

The equation for singular value decomposition of $\boldsymbol{X}$ is the following:

$$
X=U S V^{T}
$$

where $\boldsymbol{U}$ is an $m \times n$ matrix, $\boldsymbol{S}$ is an $n \times n$ diagonal matrix, and $\boldsymbol{V}^{\boldsymbol{T}}$ is also an $n \times n$ matrix. The columns of $\boldsymbol{U}$ are called the left singular vectors, $\left\{\boldsymbol{u}_{k}\right\}$, and form an orthonormal basis for the assay expression profiles, so that $\boldsymbol{u}_{i} \cdot \boldsymbol{u}_{j}=1$ for $i=j$, and $\boldsymbol{u}_{i} \cdot \boldsymbol{u}_{j}=0$ otherwise. The rows of $\boldsymbol{V}^{\boldsymbol{T}}$ contain the elements of the right singular vectors, $\left\{\boldsymbol{v}_{k}\right\}$, and form an orthonormal basis for the gene transcriptional responses. The elements of $\boldsymbol{S}$ are only nonzero on the diagonal, and are called the singular values. Thus, $\boldsymbol{S}$ $=\operatorname{diag}\left(s_{1}, \cdots, s_{n}\right)$. Furthermore, $s_{k}>0$ for $1 \leq k \leq r$, and $s_{i}=0$ for $(r+1) \leq k \leq n$. By convention, the ordering of the singular vectors is determined by high-to-low sorting of singular values, with the highest singular value in the upper left index of the $S$ matrix. Note that for a square, symmetric matrix $\boldsymbol{X}$, singular value decomposition is equivalent to diagonalization, or solution of the eigenvalue problem.
One important result of the SVD of $\boldsymbol{X}$ is that:

$$
X^{(l)}=\sum_{k=1}^{1} U_{k} S_{k} \mathrm{~V}_{k}^{T}
$$

is the closest rank-l matrix to $\boldsymbol{X}$. The term "closest" means that $X^{(l)}$ minimizes the sum of the squares of the difference of the elements of $\boldsymbol{X}$ and $X^{(l)}, \sum_{i j}\left|x_{i j}-x^{(l)}{ }_{i j}\right|^{2}$.

One way to calculate the SVD is to first calculate $V^{T}$ and $S$ by diagonalizing $\boldsymbol{X}^{T} \boldsymbol{X}$ :

$$
X^{T} X=V S^{2} V^{T}
$$

and then to calculate $\boldsymbol{U}$ as follows:

$$
\boldsymbol{U}=\boldsymbol{X V S ^ { - 1 }}
$$

where the $(r+1), \cdots, n$ columns of $\boldsymbol{V}$ for which $s_{k}=0$ are ignored in the matrix multiplication of Equation (4). Choices for the remaining $n-r$ singular vectors in $\boldsymbol{V}$ or $\boldsymbol{U}$ may be calculated using the Gram-Schmidt orthogonalization process or some other extension method. In practice there are several methods for calculating the SVD that are of higher accuracy and speed. Section 4 lists some references on the mathematics and computation of SVD.

\section{Relation between PCA and SVD}

There is a direct relation between PCA and SVD in the case where principal components are calculated from the covariance matrix. If one conditions the data matrix $\boldsymbol{X}$ by centering each column, then $\boldsymbol{X}^{\boldsymbol{T}} \boldsymbol{X}=\Sigma_{i} \boldsymbol{g}_{i} \boldsymbol{g}_{i}^{\mathrm{T}}$ is proportional to the covariance matrix of the variables of $\boldsymbol{g}_{i}$ (i.e., the covariance matrix of the assays). By Equation (3), diagonalization of $\boldsymbol{X}^{\boldsymbol{T}} \boldsymbol{X}$ yields $\boldsymbol{V}^{\boldsymbol{T}}$, which also yields the principal components of $\left\{\boldsymbol{g}_{i}\right\}$. So, the right singular vectors $\left\{\boldsymbol{v}_{k}\right\}$ are the same as the principal components of $\left\{\boldsymbol{g}_{i}\right\}$. The eigenvalues of $\boldsymbol{X}^{\boldsymbol{T}} \boldsymbol{X}$ are equivalent to $s_{k}^{2}$, which are proportional to the variances of the principal components. The matrix US then contains the principal component scores, which are the coordinates of the genes in the space of principal components.

If instead each row of $\boldsymbol{X}$ is centered, $\boldsymbol{X} \boldsymbol{X}^{T}=\Sigma_{j} \boldsymbol{a}_{j} \boldsymbol{a}_{j}^{T}$ is proportional to the covariance matrix of the variables of $\boldsymbol{a}_{j}$ (i.e. the covariance matrix of the genes). In this case, the left singular vectors $\left\{\boldsymbol{u}_{k}\right\}$ are the same as the principal components of $\left\{\boldsymbol{a}_{j}\right\}$. The $s_{k}{ }^{2}$ are again proportional to the variances of the principal components. The matrix $\boldsymbol{S} \boldsymbol{V}^{T}$ again contains the principal component scores, which are the coordinates of the assays in the space of principal components.

\section{Section 2: Application of PCA and SVD}

\section{Gene Expression}

As we mention in the introduction, gene expression data are well suited to analysis using SVD/PCA. In this section we provide examples of SVD-based analysis methods as applied to gene expression analysis. Before illustrating specific techniques, we will discuss ways of interpreting the SVD in the context of gene expression data. This interpretation and the accompanying nomenclature will serve as a foundation for understanding the methods described later.

A natural question for a biologist to ask is: "What is the biological significance of the SVD?" There is, of course, no general answer to this question, as it depends on the specific application. We can, however, consider classes of experiments and provide them as a guide for individual cases. For this purpose we define two broad classes of applications under which most studies will fall: systems biology applications, and diagnostic applications (see below). In both cases, the $n$ columns of the 
gene expression data matrix $X$ correspond to assays, and the $m$ rows correspond to the genes. The SVD of $\boldsymbol{X}$ produces two orthonormal bases, one defined by right singular vectors and the other by left singular vectors. Referring to the definitions, the right singular vectors span the space of the gene transcriptional responses $\left\{\boldsymbol{g}_{i}\right\}$ and the left singular vectors span the space of the assay expression profiles $\left\{\boldsymbol{a}_{j}\right\}$. Following the convention of Alter et al. (2000), we refer to the left singular vectors $\left\{\boldsymbol{u}_{k}\right\}$ as eigenassays and to the right singular vectors $\left\{\boldsymbol{v}_{k}\right\}$ as eigengenes.

We sometimes refer to an eigengene or eigenassay generically as a singular vector, or, by analogy with PCA, as a component. In systems biology applications, we generally wish to understand relations among genes. The signal of interest in this case is the gene transcriptional response $\boldsymbol{g}_{i}$. By Equation (1), the SVD equation for $\boldsymbol{g}_{i}$ is

$$
\boldsymbol{g}_{i}=\sum_{k=1}^{r} U_{i k} S_{k} V_{k}, i: 1, \cdots, m
$$

which is a linear combination of the eigengenes $\left\{\boldsymbol{v}_{k}\right\}$. The $i^{\text {th }}$ row of $U, \boldsymbol{g}_{i}^{\prime}$, contains the coordinates of the $i^{\text {th }}$ gene in the coordinate system (basis) of the scaled eigengenes, $s_{k} \boldsymbol{v}_{k}$. If $r<n$, the transcriptional responses of the genes may be captured with fewer variables using $\boldsymbol{g}_{i}^{\prime}$ rather than $\boldsymbol{g}_{i}$. This property of the SVD is sometimes referred to as dimensionality reduction. In order to reconstruct the original data, however, we still need access to the eigengenes, which are $n$-dimensional vectors. Note that due to the presence of noise in the measurements, $r=$ $n$ in any real gene expression analysis application, though the last singular values in $\boldsymbol{S}$ may be very close to zero and thus irrelevant.

An analysis of micro-array data is a search for genes that have similar, correlated patterns of expression. This indicates that some of the data might contain redundant information. For example, if a group of experiments were more closely related than we had expected, we could ignore some of the redundant experiments, or use some average of the information without loss of information (Khan et al., 2011; Quackenbush, 2001). Some examples are given of previous applications of SVD to analysis of gene expression data.

Cell-cycle gene expression data display strikingly simple patterns when analyzed using SVD. Here we discuss two different studies that, despite having used different pre-processing methods, have produced similar results (Alter et al., 2000; Holter et al., 2000). Both studies found cyclic patterns for the first two eigengenes, and, in two-dimensional correlation scatter plots, previously identified cell cycle genes tended to plot towards the perimeter of a disc. Alter et al. used information in SVD correlation scatter plots to obtain a result that 641 of the 784 cell-cycle genes identified in are associated with the first two eigengenes (Spellman et al., 1998). Holter et al. displayed previously identified cell-cycle gene clusters in scatter plots, revealing that cell-cycle genes were relatively uniformly distributed in a ring-like feature around the perimeter, leading Holter et al. to suggest that cell-cycle gene regulation may be a more continuous process than had been implied by the previous application of clustering algorithms (Holter et al., 2000).

Raychaudhuri et al.'s study of yeast sporulation time series data is an early example of application of PCA to microarray analysis (Raychaudhuri et al., 2000). In this study, over $90 \%$ of the variance in the data was explained by the first two components of the PCA. The first principal component contained a strong steady-state signal. Projection scatter plots were used in an attempt to visualize previously identified gene groups, and to look for structures in the data that would indicate separation of genes into groups. No clear structures were visible that indicated any separation of genes in scatter plots. Holter et al.'s more recent SVD analysis of yeast sporulation data made use of a different pre-processing scheme from that of Raychaudhuri et al. The crucial difference is that the rows and columns of $X$ in Holter et al.'s study were iteratively centered and normalized. In Holter et al.'s analysis, the first two eigengenes were found to account for over $60 \%$ of the variance for yeast sporulation data. The first two eigengenes were significantly different from those of Raychaudhuri et al., with no steady-state signal, and, most notably, structure indicating separation of gene groups was visible in the data. Below we discuss the discrepancy between these analyses of yeast sporulation data.

\section{Other Applications in Gene Expression Analysis}

Image processing and compression. The property of SVD to provide the closest rank- $l$ approximation for a matrix $\boldsymbol{X}$ (Equation (2)) can be used in image processing for compression and noise reduction, a very common application of SVD. By setting the small singular values to zero, we can obtain matrix approximations whose rank equals the number of remaining singular values (see Equation (2)). Each term $\boldsymbol{u}_{k} S_{k} \boldsymbol{v}_{k}^{T}$ is called a principal image. Very good approximations can often be obtained using only a small number of terms (Richards \& Jia, 2006). SVD is applied in similar ways to signal processing problems.

Immunology. One way to capture global prototypical immune response patterns is to use PCA on data obtained from measuring antigen-specific $\operatorname{IgM}$ (dominant antibody in primary immune responses) and $\operatorname{IgC}$ (dominant antibody in secondary immune responses) immunoglobulins using ELISA assays. Fesel and Coutinho (Fesel \& Coutinho, 1998) measured IgM and $\mathrm{IgC}$ responses in Lewis and Fischer rats before and at three time points after immunization with myelin basic protein (MBP) in complete Freud's adjuvant (CFA), which is known to provoke experimental allergic encephalomeyelitis (EAE). They discovered distinct and mutually independent components of IgM reaction repertoires, and identified a small number of strain-specific prototypical regulatory responses.

Molecular dynamics. PCA and SVD analysis methods have been developed for characterizing protein molecular dynamics trajectories (Romo et al., 1995). In a study of myoglobin, Romo et al. used molecular dynamics methods to obtain atomic positions of all atoms sampled during the course of a simulation. The higher principal components of the dynamics were found to correspond to large-scale motions of the protein. Visualization of the first three principal components revealed an interesting type of trajectory that was described as resembling beads on a string, and revealed a visibly sparse sampling of the configuration space.

Small-angle scattering. SVD has been used to detect and characterize structural intermediates in biomolecular smallangle scattering experiments (Chen et al., 1996). This study provides a good illustration of how SVD can be used to extract biologically meaningful signals from the data. Small-angle scattering data were obtained from partially unfolded solutions of lysozyme, each consisting of a different mix of folded, collapsed and unfolded states. The data for each sample was in the form of intensity values sampled at on the order of 100 different scattering angles. UV spectroscopy was used to determine the relative amounts of folded, collapsed and unfolded lysozyme in each sample. SVD was used in combination with the spectroscopic data to extract a scattering curve for the collapsed state of the lysozyme, a structural intermediate that was not observed in isolation. 
Information Retrieval. SVD became very useful in Information Retrieval (IR) to deal with linguistic ambiguity issues. IR works by producing the documents most associated with a set of keywords in a query. Keywords, however, necessarily contain much synonymy (several keywords refer to the same concept) and polysemy (the same keyword can refer to several concepts). For instance, if the query keyword is "feline", traditional IR methods will not retrieve documents using the word "cat"- a problem of synonymy. Likewise, if the query keyword is "java", documents on the topic of Java as a computer language, Java as an Island in Indonesia, and Java as a coffee bean will all be retrieved-a problem of polysemy. A technique known Latent Semantic Indexing (LSI) (Berry et al., 1995) addresses these problems by calculating the best rank-l approximation of the keyword-document matrix using its SVD. This produces a lower dimensional space of singular vectors that are called eigen-keywords and eigen-documents. Each eigen-key-word can be associated with several keywords as well as particular senses of keywords. In the synonymy example above, "cat" and "feline" would therefore be strongly correlated with the same eigen-keyterm. Similarly, documents using "java" as a computer language tend to use many of the same keywords, but not many of the keywords used by documents describing "java" as coffee or Indonesia. Thus, in the space of singular vectors, each of these senses of "java" is associated with distinct eigen-keywords.

\section{Population Genetics}

Novembre and Stephens pointed out PCA is a tool for analyzing genetic data. PCA remains useful for genetic analysis in many contexts that do not require a historical interpretation, such as in detecting the presence of population structure or in correcting for stratification in disease studies (Novembre \& Stephens, 2008). On the other hand, if the aim is to study history and document migrations, it is important to carry out additional research to correlate the PCA results with other lines of evidence.

By superimposing the PCs on the geography of the sampled populations, they obtained "synthetic maps" that showed remarkable gradients of variation across continents suggestive of historical migrations (Pearson, 1901). For example, the first European PC map shows a southeast-to-northwest cline that was interpreted as reflecting the spread of Neolithic farming from the Levant throughout Europe between 9000 and 6000 years ago. The hypothesis of a demic diffusion of Neolithic farming has since been supported by additional genetic and archaeological data (Pinhasi et al., 2005; Semino et al., 2004; Sokal et al., 1991).

\section{Population Structure and Stratification in Disease Studies}

PCA has a population genetics interpretation and can be used to partly identify differences in ancestry among populations and samples. In particular, by assessing whether the proportion of the variance explained by the first PC is sufficiently large, it is possible to obtain a formal $\mathrm{P}$ value for the presence of population substructure and to identify the number of PCs that are statistically significant (Patterson et al., 2006). PCA is also useful as a method to address the problem of population stratification-allele frequency differences between cases and controls due to ancestry differences or under selection - that can cause spurious associations in disease association studies. We and others have described how one can correct for stratification in structured populations such as European Americans by ad- justing genotypes and phenotypes by amounts attributable to ancestry along the top PCs (Price et al., 2006; Zhu et al., 2008). Novembre and Stephens (Novembre \& Stephens, 2008) emphasize that this approach is appropriate regardless of whether the PCs have arisen as a result of migrations, isolation by distance or both.

PCA is a tool that has been used to infer population structure in genetic data for several decades, long before the era of GWA studies (Novembre \& Stephens, 2008; Patterson et al., 2006; Pearson, 1901; Price et al., 2006). It should be noted that top principal components do not always reflect population structure: they may reflect family relatedness (Patterson et al., 2006), long-range linkage disequilibrium (LD) (due to, for example, inversion polymorphisms) or assay artifacts (Clayton et al., 2005). These effects can often be eliminated by removing related samples, regions of long-range LD or low-quality data, respectively, from the data used to compute principal components. In addition, PCA can highlight effects of differential bias that require additional quality control (Price et al., 2006).

Using top principal components as covariates corrects for stratification in GWA studies (Purcell et al., 2007; Zhu et al., 2008), and this can be done using software such as EIGENSTRAT. Like structured association, PCA will appropriately apply a greater correction to markers with large differences in allele frequency across ancestral populations. Unlike initial implementations of structured association, PCA is computationally tractable in large genome-wide data sets. Related approaches, such as multidimensional scaling (MDS) and genetic matching, have also proven useful (Lee et al., 2010; Luca et al., 2008) and can be carried out using the PLINK software (Purcell et al., 2007). When genome-wide data are not available (for example, in replication studies), structured association or PCA can infer genetic ancestry, and hence correct for stratification, using ancestry-informative markers (AIMs) (Furey et al., 2000). A common misconception is that AIMs should be used to infer genetic ancestry even when genome-wide data are available, but in fact the best ancestry estimates are obtained using a large number of random markers, as the examples we supplied in the following section.

\section{Qualified Inferences about Human History}

Given the results of Novembre and Stephens (2008), what confidence should we have in use of PCA for inferences regarding human history? To illustrate this, David Reich et al. turned to a dataset of 940 individuals from 53 populations typed at $\sim 650,000$ SNPs as part of the Human Genome Diversity Project( Li et al., 2008; Reich et al., 2008). They used EIGENSOFT (Patterson et al., 2006; Price, et al., 2006) to find the principal axes of genetic variation in the seven sub-Saharan African populations in this dataset and then projected all samples on the resulting PCs. Another example Quebec population study, the distribution of Mendelian diseases points to local founder effects suggesting stratification of the contemporary French Canadian gene pool. They characterized the population structure through the analysis of the genetic contribution of 7798 immigrant founders identified in the genealogies of 2221 subjects partitioned in eight regions. To detect population stratification from genealogical data, they propose an approach based on principal component analysis (PCA) of immigrant founders' genetic contributions. Results showed evidence of a distinct identity of the northeastern and eastern regions and stratification of the regional populations correlated with geographical location along the St-Lawrence River. Analysis of PC-correlated founders illustrates the differential impact of 
early versus latter founders consistent with specific regional genetic patterns. These results highlight the importance of considering the geographic origin of samples in the design of genetic epidemiology studies conducted in Quebec (Claude \& Bherer, 2011). Another example comes from a Brazil group: they used SNP data from 1129 individuals - 138 from the urban population of Sao Paulo, Brazil, and 991 from 11 populations of the HapMap Project. PCA was performed on the SNPs common to these populations, to identify the composition and the number of SNPs needed to capture the genetic variation of them. Both admixture and local ancestry inference were performed in individuals of the Brazilian sample. Then found individuals from the Brazilian sample fell between Europeans, Mexicans, and Africans. Brazilians are suggested to have the highest internal genetic variation of sampled populations. Their results indicate Brazilian sample analyzed descend from Amerindians, African, and/or European ancestors, but intermarriage between individuals of different ethnic origin had an important role in generating the broad genetic variation observed in the present-day population. Those examples highlight how PCA methods can provide evidence of important migration events. Interpreting the results to make reliable historical predictions, however, requires further genetic analysis and integration with other sources of information from archeology, anthropology, linguistics and geography.

\section{Section 3: New Extensions and Limits of PCA}

This section provides an important context for understanding when PCA might perform poorly as well as a road map for understanding new extensions to PCA, as follows:

\section{Linearity}

Linearity frames the problem as a change of basis. Several areas of research have explored how applying a nonlinearity prior to performing PCA could extend this algorithm - this has been termed kernel PCA.

\section{Mean and variance are sufficient statistics.}

The formalism of sufficient statistics captures the notion that the mean and the variance entirely describe a probability distribution. The only class of probability distributions that are fully described by the first two moments are exponential distributions (e.g. Gaussian, Exponential et al.). In order for this assumption to hold, the probability distribution of xi must be exponentially distributed. Deviations from this could invalidate this assumption.

\section{Large variances have important dynamics.}

This assumption also encompasses the belief that the data has a high SNR. Hence, principal components with larger associated variances represent interesting dynamics, while those with lower variances represent noise.

\section{The principal components are orthogonal.}

This assumption provides an intuitive simplification that makes PCA soluble with linear algebra decomposition techniques. These techniques are highlighted in the two following sections. We have discussed all aspects of deriving PCA- what remain are the linear algebra solutions. The first solution is somewhat straightforward while the second solution involves understanding an important algebraic decomposition.

\section{Limits}

Both the strength and weakness of PCA is that it is a nonparametric analysis. One only needs to make the assumptions and then calculate the corresponding answer, while it is the same on population and gene expression data analysis. There are no parameters to tweak and no coefficients to adjust based on user experience, the answer is unique and independent of the user.

This same strength can also be viewed as a weakness. If one knows a-priori some features of the structure of a system, then it makes sense to incorporate these assumptions into a parametric algorithm —or an algorithm with selected parameters.

In gene expression study, most implementations of PCA, it is difficult to define accurately the precise boundaries of distinct clusters in the data, or to define genes (or experiments) belonging to each cluster. In population genetics, a limitation of PCA is that they do not model family structure or cryptic relatedness. These factors may lead to inflation in test statistics if they are not explicitly modeled because samples that are correlated are assumed to be uncorrelated. And association statistics that explicitly account for family structure or cryptic relatedness are likely to achieve higher power owing to improved weighting of the data.

Another weakness is sometimes though the assumptions themselves are too stringent. One might envision situations where the principal components need not be orthogonal. Furthermore, the distributions along each dimension (xi) need not be Gaussian. The largest variances do not correspond to the meaningful axes; Diagonalzing a covariance matrix might not produce satisfactory results. The most rigorous form of removing redundancy is statistical independence.

$$
\mathrm{P}(\mathrm{y} 1, \mathrm{y} 2)=\mathrm{P}(\mathrm{y} 1) \mathrm{P}(\mathrm{y} 2)
$$

where $\mathrm{P}(\cdot)$ denotes the probability density. Thus PCA fails.

However, PCA is still a powerful technique for the analysis when, 1) used with another classification technique, such as $k$-means clustering or SOMs, that requires the user to specify the number of clusters. More frequently, this prior non-linear transformation is sometimes termed a kernel transformation and the entire parametric algorithm is termed kernel PCA. Other common kernel transformations include Fourier and Gaussian transformations. This procedure is parametric because the user must incorporate prior knowledge of the structure in the selection of the kernel but it is also more optimal in the sense that the structure is more concisely described. 2) This less constrained set of problems is not trivial and only recently has been solved adequately via Independent Component Analysis (ICA) (Hyv Rinen \& Oja, 2000). ICA decomposes the expression data into a set of statistically independent modes that we term as "ICA traits". The statistical independence between modes is estimated by optimizing a contrast function, such as kurtosis or mutual information (Biswas et al., 2008). Unlike SVD, ICA components might differ based on the contrast function and number of underlying sources, which under a generative model is responsible for the variation in the data.

It is important to note that application of SVD and PCA to modern anthropological genetics is relatively recent, and that methods are currently evolving. Presently, modern genetics analysis in general tends to consist of iterative applications of interactively performed analysis methods. The detailed path of any given analysis depends on what specific scientific questions are being addressed. As new inventions emerge, and further techniques and insights are obtained from other disciplines, we mark progress towards the goal of an integrated, theoretically sound approach to modern genetics.

\section{References}

Alter, O., Brown, P. O., \& Botstein, D. (2000). Singular value decom- 
position for genome-wide expression data processing and modeling. Proceedings of the National Academy of Sciences, 97, 10101. doi:10.1073/pnas.97.18.10101

Alter, O., Brown, P. O., \& Botstein, D. (2003). Generalized singular value decomposition for comparative analysis of genome-scale expression data sets of two different organisms. Proceedings of the $\mathrm{Na}$ tional Academy of Sciences, 100, 3351. doi: $10.1073 /$ pnas.0530258100

Alter, O., \& Golub, G. H. (2006). Singular value decomposition of genome-scale mRNA lengths distribution reveals asymmetry in RNA gel electrophoresis band broadening. Proceedings of the National Academy of Sciences, 103, 11828. doi:10.1073/pnas.0604756103

Berry, M. W., Dumais, S. T., \& O’Brien, G. W. (1995). Using linear algebra for intelligent information retrieval. SIAM Review, 37, 573595. doi: $10.1137 / 1037127$

Biswas, S., Storey, J., \& Akey, J. (2008). Mapping gene expression quantitative trait loci by singular value decomposition and independent component analysis. BMC Bioinformatics, 9, 244. doi:10.1186/1471-2105-9-244

Cavalli-Sforza, L. L., Menozzi, P., \& Piazza, A. (1994). The history and geography of human genes. Princeton, NJ: Princeton University Press.

Chen, L., Hodgson, K. O., \& Doniach, S. (1996). A lysozyme folding intermediate revealed by solution X-ray scattering. Journal of Molecular Biology, 261, 658-671. doi:10.1006/jmbi.1996.0491

Clayton, D. G., Walker, N. M., Smyth, D. J., Pask, R., Cooper, J. D., Maier, L. M., Smink, L. J., Lam, A. C., Ovington, N. R., \& Stevens, H. E. (2005). Population structure, differential bias and genomic control in a large-scale, case-control association study. Nature Genetics, 37, 1243-1246. doi:10.1038/ng1653

Fesel, C., \& Coutinho, A. (1998). Dynamics of serum IgM autoreactive repertoires following immunization: strain specificity, inheritance and association with autoimmune disease susceptibility. European Journal of Immunology, 28, 3616-3629.

doi:10.1002/(SICI)1521-4141(199811)28:11<3616::AID-IMMU361 6>3.0.CO;2-B

Furey, T. S., Cristianini, N., Duffy, N., Bednarski, D. W., Schummer, M., \& Haussler, D. (2000). Support vector machine classification and validation of cancer tissue samples using microarray expression data. Bioinformatics, 16, 906. doi:10.1093/bioinformatics/16.10.906

Handley, L. J. L., Manica, A., Goudet, J., \& Balloux, F. (2007). Going the distance: Human population genetics in a clinal world. TRENDS in Genetics, 23, 432-439. doi:10.1093/bioinformatics/16.10.906

Holter, N. S., Mitra, M., Maritan, A., Cieplak, M., Banavar, J. R., \& Fedoroff, N. V. (2000). Fundamental patterns underlying gene expression profiles: Simplicity from complexity. Proceedings of the National Academy of Sciences, 97, 8409. doi:10.1073/pnas.150242097

Hyv Rinen, A., \& Oja, E. (2000). Independent component analysis: Algorithms and applications. Neural Networks, 13, 411-430. doi:10.1016/S0893-6080(00)00026-5

Khan, J., Wei, J. S., Ringnér, M., Saal, L. H., Ladanyi, M., Westermann, F., Berthold, F., Schwab, M., Antonescu, C. R., \& Peterson, C. (2001). Classification and diagnostic prediction of cancers using gene expression profiling and artificial neural networks. Nature Medicine, 7, 673-679. doi:10.1038/89044

Lee, A. B., Luca, D., Klei, L., Devlin, B., \& Roeder, K. (2010). Discovering genetic ancestry using spectral graph theory. Genetic Epidemiology, 34, 51-59.

Li, J. Z., Absher, D. M., Tang, H., Southwick, A. M., Casto, A. M., Ramachandran, S., Cann, H. M., Barsh, G. S., Feldman, M., \& CavalliSforza, L. L. (2008). Worldwide human relationships inferred from genome-wide patterns of variation. Science, 319, 1100. doi:10.1126/science. 1153717

Luca, D., Ringquist, S., Klei, L., Lee, A. B., Gieger, C., \& Wichmann, H. (2008). On the use of general control samples for genome-wide association studies: genetic matching highlights causal variants. The American Journal of Human Genetics, 82, 453-463. doi:10.1016/j.ajhg.2007.11.003

Mellars, P. (2006). Going east: New genetic and archaeological perspectives on the modern human colonization of Eurasia. Science, 313, 796. doi:10.1016/j.ajhg.2007.11.003

Menozzi, P., Piazza, A., \& Cavalli-Sforza, L. (1978). Synthetic maps of human gene frequencies in Europeans. Science, 201, 786. doi:10.1126/science. 356262

Novembre, J., \& Stephens, M. (2008). Interpreting principal component analyses of spatial population genetic variation. Nature Genetics, 40, 646-649. doi:10.1038/ng.139

Omberg, L., Golub, G. H., \& Alter, O. (2007). A tensor higher-order singular value decomposition for integrative analysis of DNA microarray data from different studies. Proceedings of the National Academy of Sciences, 104, 18371. doi:10.1073/pnas.0709146104

Patterson, N., Price, A. L., \& Reich, D. (2006). Population structure and eigenanalysis. PLoS Genetics, 2, e190. doi:10.1371/journal.pgen.0020190

Pearson, K. LIII. (1901). On lines and planes of closest fit to systems of points in space. Philosophical Magazine Series 6, 2, 559-572. doi:10.1080/14786440109462720

Pinhasi, R., Fort, J., \& Ammerman, A. J. (2005). Tracing the origin and spread of agriculture in Europe. PLoS Biology, 3, e410. doi:10.1371/journal.pbio.0030410

Price, A. L., Patterson, N. J., Plenge, R. M., Weinblatt, M. E., Shadick, N. A., \& Reich, D. (2006). Principal components analysis corrects for stratification in genome-wide association studies. Nature, 38, 904909.

Purcell, S., Neale, B., Todd-Brown, K., Thomas, L., Ferreira, M. A., Bender, D., Maller, J., Sklar, P., de Bakker, P. I., Daly, M. J., \& Sham, P. C. (2007). PLINK: A tool set for whole-genome association and population-based linkage analyses. American Journal of Human Genetics, 81, 559-575. doi:10.1086/519795

Quackenbush, J. (2001). Computational analysis of microarray data. Nature Reviews Genetics, 2, 418-427. doi:10.1038/35076576

Raychaudhuri, S., Stuart, J. M., \& Altman, R. B. (2000). Principal components analysis to summarize microarray experiments. Application to Sporulation Time Series, 455.

Reich, D., Price, A. L., \& Patterson, N. (2008). Principal component analysis of genetic data. Nature Genetics, 40, 491-491. doi:10.1038/ng0508-491

Richards, J. A., \& Jia, X. (2006). Remote sensing digital image analysis: An introduction. Berlin: Springer Verlag.

Romo, T. D., Clarage, J. B., Sorensen, D. C., \& Phillips Jr, G. N. (1995). Automatic identification of discrete substates in proteins: Singular value decomposition analysis of time-Averaged crystalllographic refinements. Proteins: Structure, Function, and Bioinformatics, 22, 311-321. doi:10.1002/prot.340220403

Semino, O., Magri, C., Benuzzi, G., Lin, A. A., Al-Zahery, N., Battaglia, V., Maccioni, L., Triantaphyllidis, C., Shen, P., \& Oefner, P. J. (2004). Origin, diffusion, and differentiation of Y-chromosome haplogroups $\mathrm{E}$ and $\mathrm{J}$ : Inferences on the neolithization of Europe and later migratory events in the Mediterranean area. The American Journal of Human Genetics, 74, 1023-1034. doi:10.1086/386295

Sokal, R. R., Oden, N. L., \& Wilson, C. (1991). Genetic evidence for the spread of agriculture in Europe by demic diffusion. Nauture, 351, 143-145. doi:10.1038/351143a0

Spellman, P. T., Sherlock, G., Zhang, M. Q., Iyer, V. R., Anders, K., Eisen, M. B., Brown, P. O., Botstein, D., \& Futcher, B. (1998). Comprehensive identification of cell cycle-regulated genes of the yeast Saccharomyces cerevisiae by microarray hybridization. Molecular Biology of the Cell, 9, 3273.

Yeung, K. Y., \& Ruzzo, W. L. (2001). Principal component analysis for clustering gene expression data. Bioinformatics, 17, 763 . doi:10.1093/bioinformatics/17.9.763

Zhu, X., Li, S., Cooper, R. S., \& Elston, R. C. (2008). A unified association analysis approach for family and unrelated samples correcting for stratification. The American Journal of Human Genetics, 82, 352365. doi:10.1016/j.ajhg.2007.10.009 